\title{
Robust Prognostics Concept for Gearbox with Artificially Induced Gear Crack Utilizing Acoustic Emission
}

\author{
Shawki A. Abouel-seoud \\ Department of Automotive Engineering, Helwan University, Cairo, PO box 11718, Egypt \\ Tel: 2-100-465-1521 E-mail: s_a_seoud@hotmail.com \\ Mohamed S. Elmorsy \\ Department of Mechanical Design, Helwan University, Cairo, PO box 11718, Egypt \\ Tel: 2-100-682-4670Ｅ-mail: Mohsabry1999@yahoo.com \\ Eid S. Dyab \\ Department of Automotive Engineering, Helwan University, Cairo, PO box 11718, Egypt \\ Tel: 2-100-642-7775 E-mail: eng_eid74@yahoo.com
}

\begin{abstract}
Received: October 20, 2011 Accepted: October 27, 2011 Published: December 31, 2011
doi:10.5539/eer.v1n1p81 URL: http://dx.doi.org/10.5539/eer.v1n1p81
\end{abstract}

\begin{abstract}
Prognostic is a rapidly developing field and seeks to build on current diagnostic equipment capabilities for predicting the system state in advance. In machine condition prognostics, the current and past observations are used to predict the upcoming states of the machine. Signal-de-noising and extraction of the weak acoustic signature are crucial to gearbox prognostics since the inherent deficiency of the measuring mechanism often introduces a great amount of noise to the signal. In addition, the signature of a defective gearbox is spread across a wide frequency band and hence can easily become masked by noise and low frequency effects. As a result, robust concepts are needed to provide more evident information for gearbox performance assessment and prognostics.

This paper introduces enhanced and robust prognostic concepts for gear tooth based on an optimal wavelet filter method for fault identification and a statistical method for performance degradation assessment. The experimental results demonstrate that the gear tooth defect can be detected and evaluated at an early stage of development when both the optimal wavelet filter and statistical analysis technique are used.
\end{abstract}

Keywords: Prognostics, Hazard rate, Reliability, Optimization, Gearbox crack, Acoustic emission, Wavelet

\section{Introduction}

\subsection{General}

Gearbox failure is one of the foremost causes of breakdowns in rotating machinery and such failure can be catastrophic, resulting in costly down time. One of the key issues in gearbox prognostics is to detect the defect at its incipient stage and alert the operator before it develops into catastrophic failure. Accurate and reliable decision making using prognostics has a very important role in effective utilization and maintenance of machinery systems. Various temporal patterns including oil debris analysis, acoustic and vibration signals, and temperature may be used for the prediction of machine condition. Among these, acoustic-based monitoring and prognostic approach is quite common in condition-based maintenance (CBM) of machines due to the ease of acoustic measurement and analysis (Hardman, et al., 1999; Jardin, et al., 2006).

To date, there has been neither an attempt toward understanding the mechanisms for generating acoustic emission (AE) activity in helical gears, nor the ability of AE to identify defects in such gears; considering helical gears are a major component of gearbox applications worldwide this is rather surprising. It is also known that the meshing mechanisms for a helical gear is progressive due to the gradual increase and decrease in contact length 
over a particular tooth whilst the spur gear mesh has a constant contact length throughout the gear mesh. Few works are reported in literature that assess the effectiveness of AE monitoring techniques for identification of seeded defects on helical gears or exploring the effect of gradual defect growth (Tan and Mba, 2005; Eftekharnejad and Mba 2009).

The interest for applications of acoustic emission (AE) for monitoring working condition in rotating machinery is relatively new and has grown significantly over the last decade. AE in rotating machinery is defined as elastic waves generated by the interaction of two media in motion, i.e. a pair of gears. Sources of AE in rotating machinery include asperities contact, cyclic fatigue, friction, material loss, cavitations, leakage, etc. AE technique has drawn attention as it offers some advantages over classical vibration monitoring. First of all, as AE is a non-directional technique, one $\mathrm{AE}$ sensor is sufficient in contrast to vibration monitoring which may require information from three axes. Since AE is produced at microscopic level it is highly sensitive and offers opportunities for identifying defects at an earlier stage when compared to other condition monitoring techniques. As AE mainly detects high-frequency elastic waves, it is not affected by structural resonances and typical mechanical background noise (under $20 \mathrm{kHz}$ ). Finally, AE was proved superior over vibration data on early detection of small defects in gears (Wang, et al., 2009; Loutas, et al., 2009)

\subsection{Background of gearbox prognostics}

The vibration or acoustic signal of a defective gearbox usually considers being amplitude modulated at characteristic defect frequency. Matching the measured vibration or noise spectrum with the defect characteristic frequency enables us to detect the presence of a defect and determine where the defect is. A traditional diagnostic scheme can be described as in Figure 1.

Driving by the desire of improved gearbox uptime and near-zero breakdown productivity, more and more attention has been put onto predictive maintenance, which necessitates advanced tools in prognostics. Prognostics is using predictive maintenance practices and tools to analyze the trends of machine performance against known engineering limits for the purpose of detecting, analyzing and correcting problem before failure occurs. More advanced prognostics are focused on performance degradation monitoring and assessment so failure can be predicted and prevented (Lin and Zuo, 2003). To fulfill the goal of prognostics, three crucial steps are needed. At first, the defect or abnormality should be able to be detected at its early stage. Secondly, the machine or system performance needs to be assessed robustly and tracked continuously. Finally, a prediction with confidence interval needs to be generated estimating the remaining useful life and possible failure mode of the machine or system. A new prognostic scheme can be depicted as shown in Figure 2.

The challenge of gearbox prognostics is how to effectively evaluate the system performance based on the extracted features. One of the primary difficulties for effective implementation of gearbox prognostics is the highly stochastic nature of defect growth. Even though a large variety of features can be extracted to describe the characteristics of acoustic emission signal from different aspects, such as RMS, Kurtosis, etc. previous research work has shown that each feature is only effective for certain defect at certain stage. For example, spikiness of the acoustic emission signals indicated by Kurtosis implies incipient defects, whereas the high energy level given by the value of RMS indicates severe defects (Xi F and Krishnappa, 2000). A good performance assessment method should take advantage of mutual information from multiple features for system degradation (hazard) assessment. Feature map is one of widely used performance degradation (hazard) assessment methods. Some successful applications can be found in ( $\mathrm{Lin}$ and $\mathrm{Qu}, 2000$ ). It is based on the assumption that if an exclusive feature space could be established using physical models, expert experiences, and/or historical data, different fault modes should be able to find its corresponding mapping coordinates on it. With the exception of abrupt catastrophic failures, most of the faults have some kind of progression to failure. Consequently their coordinates in feature space should have traceable trajectories drifting from the normal operation region to various fault regions. By continuously tracking the trajectories, degradation detection and performance assessment in feature space can be accomplished. The challenge is how to construct a feature space that can consistently exemplify the degradation pattern.

This paper introduces enhanced and robust prognostic concepts for gear tooth monitoring; a wavelet filter based method is used for weak acoustic emission enhancement and fault identification, while a statistical analysis technique based method is used for the performance degradation (hazard) assessment.

\section{The Continuous Wavelet Transform}

\subsection{Definition}

Wavelet transforms are inner products between signals and the wavelet family, which are derived from the mother 
wavelet by dilation (scale) and translation. Let $g(\mathrm{t})$ be the mother wavelet, the daughter wavelet will be $\boldsymbol{g}_{\mathrm{a}, \mathrm{b}}(\mathrm{t})=$ $\boldsymbol{g}((\mathrm{t}-\mathrm{b}) / \mathrm{a})$, where $a$ is the scale parameter and $b$ is the time translation. By varying the parameters $a$ and $b$, different daughter wavelets can be obtained that constitute a wavelet family. Wavelet transform is to perform the following operation (Lin and Zuo, 2003; Staszewski and Tomlinson, 1994):

$$
W_{g}(a ; b)=\frac{1}{\sqrt{a}} \int_{-\infty}^{+\infty} x(t) g_{a, b}^{*}(t) d t
$$

Where: $W_{\mathrm{g}}(\mathrm{a}, \mathrm{b})$ is the function after transformed, and $\boldsymbol{g} *(\mathrm{t})$ stands for complex conjugation of $g(\mathrm{t})$. For the function $g(t)$ to qualify as an analyzing wavelet, it must satisfy the admissibility conditions.

$$
c_{q}=\int_{-\infty}^{+\infty} \frac{|G(\omega)|}{|\omega|}
$$

Where $\mathrm{G}(\omega)$ is Fourier transform of $g(\mathrm{t})$. This is needed in obtaining the inverse of the wavelet transform.

If a daughter wavelet is viewed as a filter, wavelet transform is simply a filtering operation. Usually, reconstructing the wavelet coefficients at selected scales through certain methods attempted to be discovered. To do this, prior information had to be known on the signal needs to be identified.

\subsection{Morlet wavelet filter}

Morlet wavelet is one of the most popular non-orthogonal wavelets. The definition of Morlet is:

$$
\psi(t)=\exp \left(-\frac{\beta^{2} t^{2}}{2}\right) \cos (\pi t)
$$

It is a cosine signal that decays exponentially on both the left and the right sides. This feature makes it very similar to an impulse. It has been used for impulse isolation and mechanical fault diagnosis through the performance of a wavelet de-noising procedure (Lin and Zuo (2003)). However, in practice, it is not easy to provide a proper threshold for wavelet de-noising. This is can be avoided by using an adaptive wavelet filter instead of wavelet de-noising.

\subsection{Adaptive Morlet Wavelet Filter}

A daughter Morlet wavelet is obtained by time translation and scale dilation from the mother wavelet, as shown in the following formula (Qiu, et al., 2003):

$$
\psi_{a, b}(t)=\psi\left(\frac{t-b}{a}\right)=\exp \left[-\frac{\beta^{2}(t-b)^{2}}{2 a^{2}}\right] \cos \left[\frac{\pi(t-b)}{a}\right]
$$

Where $\mathrm{a}$ is the scale parameter for dilation and $\mathrm{b}$ is the time translation. It can also be looked at as a filter.

To identify the immersed impulses by filtering, the location and the shape of the frequency band corresponding to the impulses must be determined first. Scale a and parameter $\beta$ control the location and the shape of the daughter Morlet wavelet respectively. As a result, an adaptive wavelet filter could be built by optimizing the two parameters for a daughter wavelet.

Several researchers have reported on how to select the mother wavelet that adapts the best to the signal to be isolated. The methods of $(\beta)$ selection in Morlet wavelet are based on either maximum kurtosis or minimum entropy value, where the entropy is the conservation of energy (equation 6). Details on how to select $\beta$ and a in Morlet wavelet based on kurtosis to make the mother wavelet match the signal to be isolated are provided in (Qiu, et al., 2003). In this analysis, focus has been made on finding the best wavelet filter (the daughter wavelet of a Morlet wavelet) instead of optimal wavelet reconstruction.

The difference between single- and double-sided Morlet wavelet has been studied in (Qiu, et al., 2003). Their frequency spectra are quite different. Since a real impulse is usually non-symmetric, single side Morlet wavelet, using the right-hand side of as the basis, should match the behaviors of hidden impulses the best.

Kurtosis is used in engineering for detection of fault symptoms because it is sensitive to sharp variant structures, such as impulses (Qiu, et al., 2003). The bigger impulse in signals shows the larger kurtosis. As a result, kurtosis can be used as the performance measure of a Morlet wavelet filter. The definition of kurtosis is:

$$
\operatorname{Kurt}(x)=E\left(x^{4}\right)-3\left[E\left(x^{2}\right)\right]^{2}
$$


Where $x$ is the sampled time series, E represents the mathematical expectation of the series and Kurt (x) is kurtosis of the signal. The procedure to perform the adaptive wavelet filtering is as follows:

- Vary the parameters a and $\beta$ within pre-selected intervals to produce different daughter wavelets.

- Perform wavelet filtering using each daughter wavelet and calculate the kurtosis of each outcome.

- Compare the kurtosis value. The parameters $a$ and $b$ that correspond to the largest kurtosis are the best parameters to use to reveal the hidden impulses.

\section{High Order Statistics}

In a more advanced approach of time-domain analysis, time series models are applied to waveform data. The main idea of time series modeling is to fit the waveform data to a parametric time model and extract features based on this parametric model. The popular models used in the literature are the autoregressive (AR) model and the autoregressive moving average (ARMA) model (Kramberger, et al., 2004). In these models, only high order statistics of root mean square (RMS), crest factor and kurtosis are used. These features are usually called timedomain features:

1) RMS is a kind of average of signal, for discrete signals, the RMS value is defined as:

$$
\begin{aligned}
& R M S=\sqrt{\frac{1}{N} \sum_{n=1}^{N}(x(n)-\bar{x})^{2}} \\
& \bar{x}=\frac{1}{N} \sum_{n=1}^{N} x(n)
\end{aligned}
$$

2) Kurtosis is a parameter that is sensitive to the shape of the signal and is well adapted to the impulse nature of the simulating forces generated by component damage. Its value can be given by:

$$
\text { Kurtosis }=\frac{(1 / N) \sum_{n=1}^{N}(x(n)-\bar{x})^{4}}{\left[(1 / N) \sum_{n=1}^{N}(x(n)-\bar{x})^{2}\right]^{2}}
$$

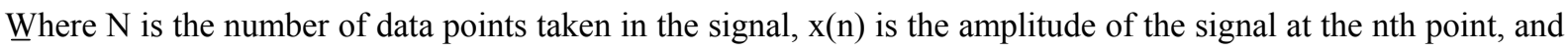
$x$ is the mean value of all the amplitudes.

3) The crest factor is defined as the ratio of the peak value to the RMS of the signal. The factor corresponds to the ratio between the crest value (maximum absolute value reached by the function representative of the signal during the considered period of time) and the root mean square (RMS) value (efficient value) of the signal.

$$
\begin{gathered}
\text { Crest Factor }=\frac{\text { Peak }}{R M S}=\frac{\text { Crest Value }}{R M S \text { Value }}=\frac{\operatorname{Sup}|x(n)|}{\sqrt{\frac{1}{N} \sum_{n=1}^{N}[x(n)]^{2}}} \\
\text { Peak }=\text { Crest Value }=\frac{1}{2}[\max (x(t))-\min (x(t))]
\end{gathered}
$$

Where $N$ is the number of samples taken within the signal and $x(\mathrm{n})$ is the time domain signal.

\section{Technical Approach}

A prognostic system in terms of remaining lifetime output that only reporting a specific time-to-failure without having any confidence bound associated with the prediction would be unwise. This is true for simple prognostic approaches that only utilize historical reliability data (such as Weibull distributions) to the more advanced prognostic modeling approaches that take design parameter and operating condition uncertainties into account. The data-driven prognostic modeling approach implemented in this paper takes advantage of the directly sensed parameter together with the historical reliability data to provide critical inputs for producing accurate failure predictions. Information from noise in terms of sound pressure level (SPL) data measurements to represent gear's fault with high certainty are used (Ireson, et al., 1996; Burdic, 1991).

The probability distribution function (pdf) can be described mathematically by $f(x)$, then the cumulative distribution function $F(x)$ can drive by continuous integration as the following 


$$
F(x)=\int_{0}^{t} f(x) d x .
$$

Whereas the sum of the reliability and the cumulative distribution function is equal to one then the equation can be written as

$$
R(x)+F(x)=1
$$

Where

$\mathrm{f}(\mathrm{x})$ : is the probability distribution function (time, kurtosis, RMS, ...etc)

$\mathrm{F}(\mathrm{x})$ : is the cumulative distribution function (time, kurtosis, RMS, ...etc)

$\mathrm{R}(\mathrm{x})$ : is the reliability function (time, kurtosis, RMS, ...etc)

The rate at which failures occur in a certain time interval $(x, x+1)$ is called the failure rate during that interval (Figure 3). It is defined as the probability that a failure per unit time occurs in the interval, given that a failure has not occurred prior to $\mathrm{x}$, the beginning of the interval. Thus the failure rate (hazard rate) is

$$
\begin{gathered}
h(x)=\frac{\int_{t}^{t+1} f(x) d x}{R(x)}=\frac{f(x)}{R(x)} \\
\int_{t}^{t+1} f(x) d t=f(x)
\end{gathered}
$$

It will be seen that if $\mathrm{dx}$ is equal to 1 and the height of the curve is assumed to be height $\mathrm{f}(\mathrm{x})$ between $\mathrm{x}$ and $\mathrm{x}+1$. That means, when the decision maker obtains the probability distribution function from the actual data for any system, he can derive the hazard function or the measured degradation of it. On the other hand, after knowing the measured degradation of the system, the remaining useful lifetime of it can be predicted.

In this paper, the failure rate is assumed to occur according to Weibull distribution function. Then the probability distribution function is

$$
f(x)=\frac{\beta(x)^{\beta-1}}{\eta^{\beta}} \exp \left[-\left(\frac{x}{\eta}\right)^{\beta}\right]
$$

From eq. (5), then

$$
F(x)=1-\exp \left[-\left(\frac{x}{\eta}\right)^{\beta}\right]
$$

From eq. (6), eq. (7)

The hazard rate given by

$$
h(x)=\frac{\beta}{\eta}\left(\frac{x}{\eta}\right)^{\beta-1}
$$

Where

$\mathrm{x}$ : is the failure threshold value (time, kurtosis, RMS,...etc)

$\eta$ : is the characteristic life or is the scale parameter.

$\beta$ : is the shape parameter

\section{Experimental Methodology}

\subsection{Experimental setup}

Figure 4 shows the experimental setup used for the gearbox testing. The gearbox consists of two gears made with a module of $3 \mathrm{~mm}$, pressure angle $20^{\circ}$, which have 64 and 26 teeth with $7 \mathrm{~mm}$ face width. The axes of the gears are supported by two ball bearings each. The entire system is settled in an oil basin in order to ensure proper 
lubrication. The gearbox is powered by electric motor and consumes its power on a hydraulic disc brake. The shafts are ball bearing supported. The system characteristics are as follows:

- 1 stage gearbox with two gears (26 and 64 teeth)

- 3-phase $5 \mathrm{hp}$ motor (220 V, 9 A, $50 \mathrm{~Hz}, 1400 \mathrm{rpm}$ ) inverter controlled

- Hydraulic disc brake

One non-destructive technique has been employed to monitor the gearbox during operation, namely acoustic emission. Measurements of acoustic in terms of sound pressure levels (SPLs) signals were carried out, see Figure 5. The Bruel \& Kjaer $(B \& K)$ portable and multi-channel PULSE type 3560-B-X05 with condenser 1/2-in microphone and preamplifier type 4189A-021. The B\&K PULSE lab shop with measurement software type 7700 was used to analyze the results. Recordings were carried out at constant speed condition and closed windows. The sampling frequency used was $1.6 \mathrm{kHz}$ and signals of $1 \mathrm{~s}$ duration were recorded.

\subsection{Signal processing}

Significant effort was dedicated to the signal processing of the acoustic waveforms acquired during the tests. The goal set a priori was to calculate a number of parameters-features extracted by the signals and check their behavior during the tests. This is done in order to identify the most promising signals that may be used for damage detection and condition monitoring of the gear system.

\subsection{Test procedure}

Many tests were conducted in order to insure the repeatability of the recordings. Generally in gearbox failure, a crack is developing at the root of a single tooth of the gear. A tooth root crack typically starts at the point of the largest stress in the material. A computational model which applies the principles of linear elastic fracture mechanics could be used to simulate gear tooth root crack propagation (Kramberger, et al., 2004). Based on the computational results, the crack propagation path shows a slight curve extending from the tooth root as shown in Figure 6. Also, indicates that crack propagation paths are smooth, continuous and in the most cases, rather straight with only a slight curvature. In this paper, according to the results shown in (Kramberger, et al., 2004), the gear crack was further simplified to consider the crack path to be a straight line as shown in Figure 6, where the crack starts at the root of the gear and then proceeds further.

In terms of various parameters evolution during the test, a representative test on a gear system with a cut of tooth root thickness (Figure 6) will be presented in this study. Many tests were conducted on the same configuration yield similar parameters behavior. A small crack was made artificially with wire electrical discharge machining at the root of gear one tooth to create a stress concentration which eventually led to a propagating crack. Figures 7 to 9 depict the gear in its health condition, a sketch of the crack dimensions for the defect (crack) at tooth root of $3.0 \times 0.2 \times 40 \mathrm{~mm}$ and the defected gear respectively. The size of cracks is a little bigger than one can encounter in the practical situation. The vibration signal from the accelerometers which are mounted on the test bearing was taken after allowing initial running of the bearing for some time.

Recordings every $1.0 \mathrm{hr}$ were acquired and a total of 7 recordings ( $\sim 6.0 \mathrm{~h}$ of test duration) were resulted until the termination of the test. This type of test was preferred in order to have the opportunity to monitor bath damage modes, i.e., the natural crack propagation. Bearing in mind, the gear was not run to failure.

\section{Results and Discussion}

Figures 10 and 11 illustrate the measured sound pressure levels in terms of time-domain signals for faulty (cracked) gear when the testing times are 0.0 hours and 6.0 hours respectively based on the experimental methodology presented above. The speed, load and crack depth are being $400 \mathrm{rpm}, 15 \mathrm{Nm}$ and $3.0 \mathrm{~mm}$ respectively. First, consider the gearbox signals are stationary waveform, and second consider the signals are non-stationary waveform. In both cases, the high order statistics presented in equations (6) to (8) were computed.

No periodic impulses appear on the waveforms even though crack may have appeared in the gear already. The periodic impulses of crack are hidden in the signal. Morlet wavelet is used to obtain the adaptive filter. Figure 12 shows the kurtosis distribution for different values of beta $(\beta)$ and scale. The parameter $\beta$ varies from 0.1 to 4.0 with step size of 0.1 and the scale varies from 1.0 to 30.0 with step size of 1.0. To illustrate the sensitivity of the kurtosis as a function of $\beta$, the value of scale a was fixed to be 2.0 and plot the kurtosis- $\beta$ relationship, which follows that the kurtosis is very sensitive to the value of $\beta$ (see Figure 13), where the largest kurtosis's value calculated based on equation (5), which is 7.75 at $\beta=0.5$ and the scale value equals to 2.0. Figure 14 shows the wavelet map where the distortion in color is nearly repeated each 600 samples depending on crack location.

The filtering results with the Morlet optimized wavelet filter $(\beta=0,5$ and $\mathrm{a}=2)$ based on equation (4). Periodic 
impulses are obvious in Figure 14. Since the speed is $400 \mathrm{rpm}$, the time considered the measurements is $500 \mathrm{~ms}$ $(0.5 \mathrm{~s})$ and the gear teeth number is 64 teeth, then the periodic is equal to one divided by speed $(\mathrm{c} / \mathrm{s})$ and is just about $0.150 \mathrm{~s}$, while the meshing frequency is calculated as gear teeth number divided by periodic and is equal to $426 \mathrm{~Hz}$.

The aforementioned equations (6) to (8) for RMS, kurtosis and crest factor high order statistics respectively are applied to the signal in filtered and unfiltered forms, where their results are shown in Figures 15 to 17, the results indicate that the use of such statistics can help to detect the gear fault growth. Furthermore, the kurtosis value is found to be a better indicator as compared to RMS or crest factor. Therefore, kurtosis is to be used as an indicator for prognostic process.

Figures 18 and 19 show the relationship between the testing time and kurtosis parameter for the measured SPL signals in the form of unfiltered and filtered based on equation (14), where Figures 20 and 21 show the relationship between hazard rate and Kurtosis for the signal in the form of unfiltered and filtered based on equation (14), where the values of the Weibull distribution parameters $(\eta, \beta)$ are 3500 and 3.2 respectively. Figures 22 and 23 illustrate the relationship between hazard rate and testing time in filtered and unfiltered forms respectively from which the remaining life can be extracted and obtained.

\section{Conclusions}

1) The experimental methodology developed in this work was utilized for diagnostic regime. Furthermore, the obvious periodical impulses caused by the cracked tooth appear in the time domain signal which carries diagnostic information. This information was found to be important for extracting features of tooth crack damage.

2) A single-stage gearbox was utilized in order to study the development of damage in artificially induced cracks in the gear. Multi-hour tests were conducted and recordings were acquired using sound pressure level monitoring, where the high order statistics of RMS, Kurtosis and crest factor reflect in the sound pressure level responses of the gearbox.

3) In non-stationary vibration waveform feature and in the process of feature extraction, it is required that the time-scale structure of a wavelet should be consistent with the feature components. For a Morlet, its time and frequency resolution can be altered by adjusting the value of $\beta$. Morlet wavelet can be used for impulse detection due to its similarity to an impulse. Any wavelet can be viewed as a filter. To identify impulses hidden in noisy signals by filtering the wavelet should well match the time-frequency structure of the impulse. An adaptive Morlet wavelet filter based on the kurtosis maximization is proposed to detect periodic impulses automatically for recognition of gear tooth crack.

4) The ability to predict the geared system remaining life time to conditional or mechanical failure (on a real-time basis) is of enormous benefit and health management systems that can effectively implement. The capabilities presented herein offer a great opportunity in terms of reducing overall life cycle costs of the geared systems as well as decreasing the operations/maintenance logistic footprint.

\section{References}

Burdic, W. S. (1991). Underwater acoustic system analysis. ( $2^{\text {nd }}$ ed.), NJ: Prentice-Hall, Englewood Cliffs (pp. 262-264).

Eftekharnejad B. and Mba, D. (2009). Seeded fault detection on helical gears with acoustic emission. Applied Acoustics, 70, 547-555. http://dx.doi.org/10.1016/j.apacoust.2008.07.006

Hardman, W., Hess, A. and sheaffer, j. (1999). SH-60 helicopter integrated diagnostic System (HIDS) program -diagnostic and prognostic development experience. Proceedings of the IEEE aerospace Conference. Aspen, CO, USA, March 6-13, 473.

Ireson, G. W, Coombs,C. F. and Moss, R. Y. (1996). Handbook of reliability engineering and Management. $\left(^{\text {nd }}\right.$ ed.), McGraw-Hill.

Jardin, A. K. S., Lin, D. and Banjevic, D. (2006). A review on machinery diagnostics and prognostics implementing condition-based maintenance. Mechanical Systems and Signal Processing, 20, 1483-1510. http://dx.doi.org/10.1016/j.ymssp.2005.09.012

Kramberger, J., Sraml, M., Glodez, S. Flasker, J. and Potrc, I. (2004). Computational model for the analysis of bending fatigue in gears. Computers and structures, 82(23-26), 2261-2269. http://dx.doi.org/10.1016/j.compstruc. 2003.10.028 
Lin, J. and Qu, L. (2000). Feature extraction based on Morlet wavelet and its application for fault diagnosis. Journal of Sound and Vibration, 234(1), 135-138. http://dx.doi.org/10.1006/jsvi.2000.2864

Lin, J. and Zuo, M. (2003). Gearbox fault diagnosis using adaptive wavelet filter. Mechanical Systems and Signal Process, 17(6), 1259-1269. http://dx.doi.org/10.1006/mssp.2002.1507

Loutas, T. H., Sotiriades, G., Kalaitzoglou, I. and Kostopoulos, V. (2009). Condition monitoring of a single-stage gearbox with artificially induced gear cracks utilizing on- line vibration and acoustic emission measurements. Applied Acoustics, 70, 1148-1159. http://dx.doi.org/10.1016/j.apacoust.2009.04.007

Qiu, H., Lee, J., Lin, J and Yu, G. (2003). Robust performance degradation assessment methods for enhanced rolling element bearing prognostics. Advanced Engineering Informatics, 17, 127-140. http://dx.doi.org/10.1016/j. aei.2004.08.001

Staszewski, W. J. and Tomlinson, G. R. (1994). Application of the wavelet transform to fault detection in a spur gear. Mechanical Systems and Signal Processing, 8(3), 289-307. http://dx.doi.org/10.1006/mssp.1994.1022

Tan, C. K. and Mba, D. (2005). Limitation of acoustic emission for identifying seeded defects in Gearboxes. Journal of Non-Destructive Evaluation, 24 (1), 11-28. http://dx.doi.org/10.1007/s10921-005-6657-9

Wang, D., Miao, Q. and Kang, R. (2009). Robust health evaluation of gearbox subject to tooth failure with wavelet decomposition". Journal of Sound and Vibration, 324, 1141-1157. http://dx.doi.org/10.1016/j.jsv.2009.02.013

Xi F, S. Q. and Krishnappa G. (2000). Bearing diagnostics based on pattern recognition of statistical parameters. Journal of Vibration Control, 6, 375-392. http://dx.doi.org/10.1177/107754630000600303

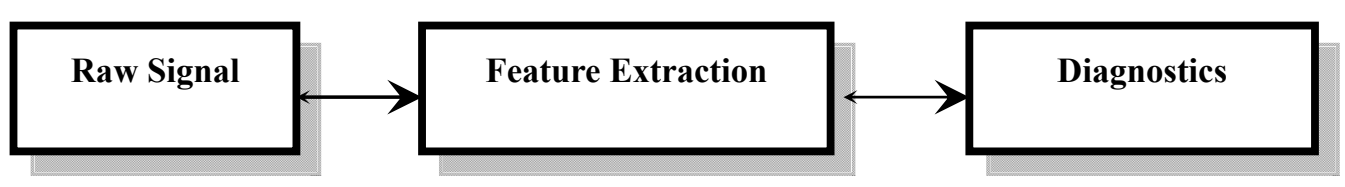

Figure 1. Traditional gearbox diagnostics scheme

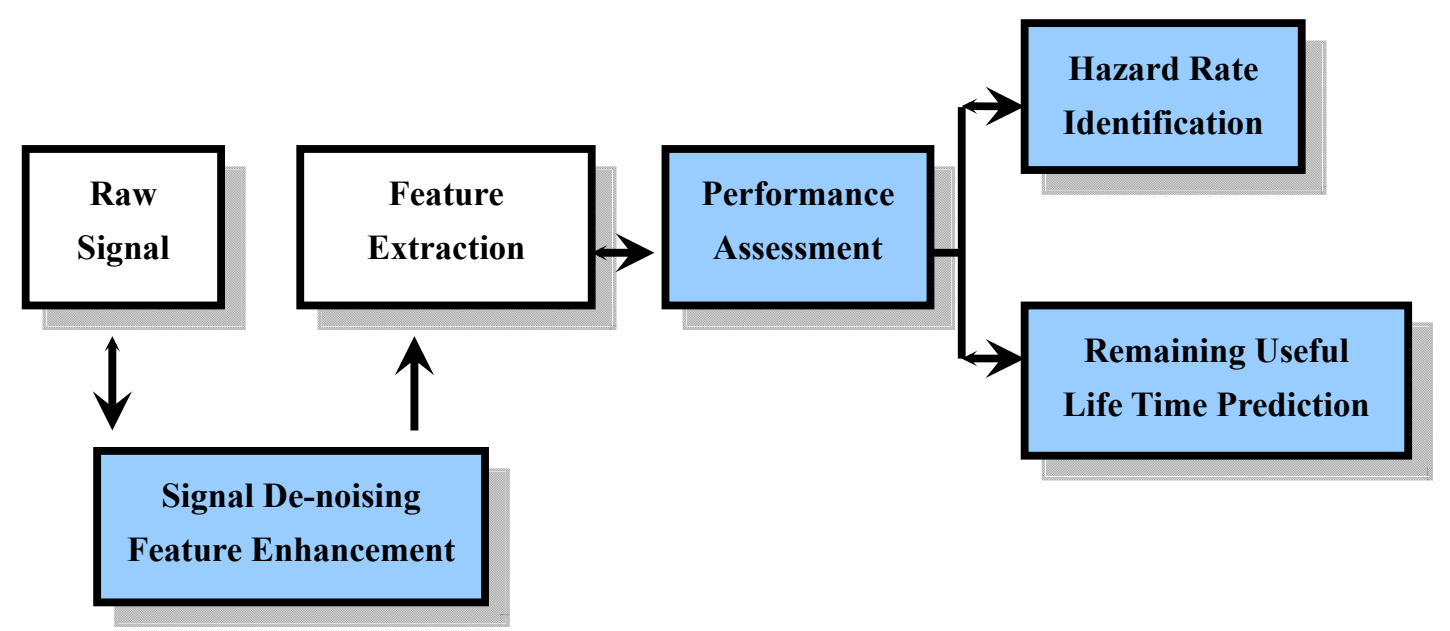

Figure 2. Proposed prognostic scheme 


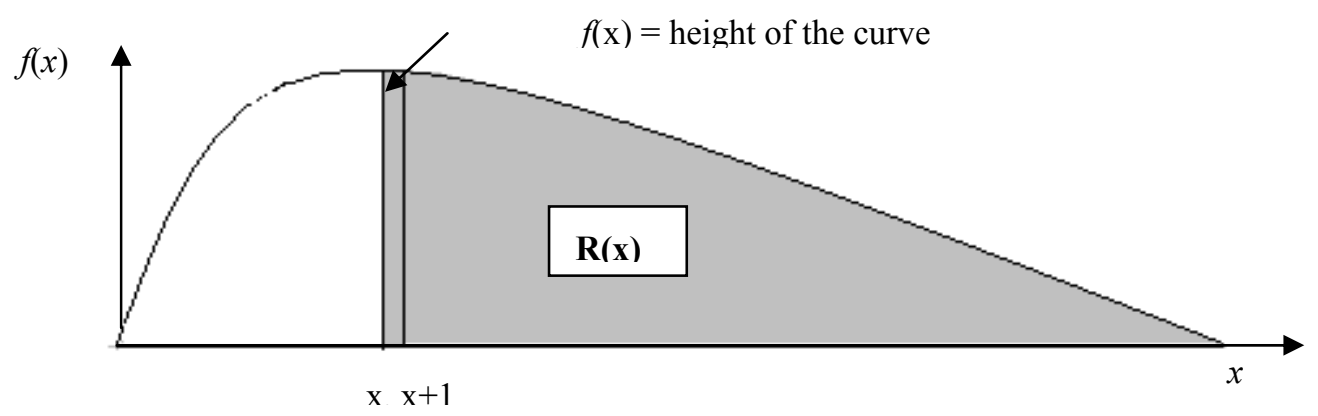

Figure 3. Derivation of Failure Rate

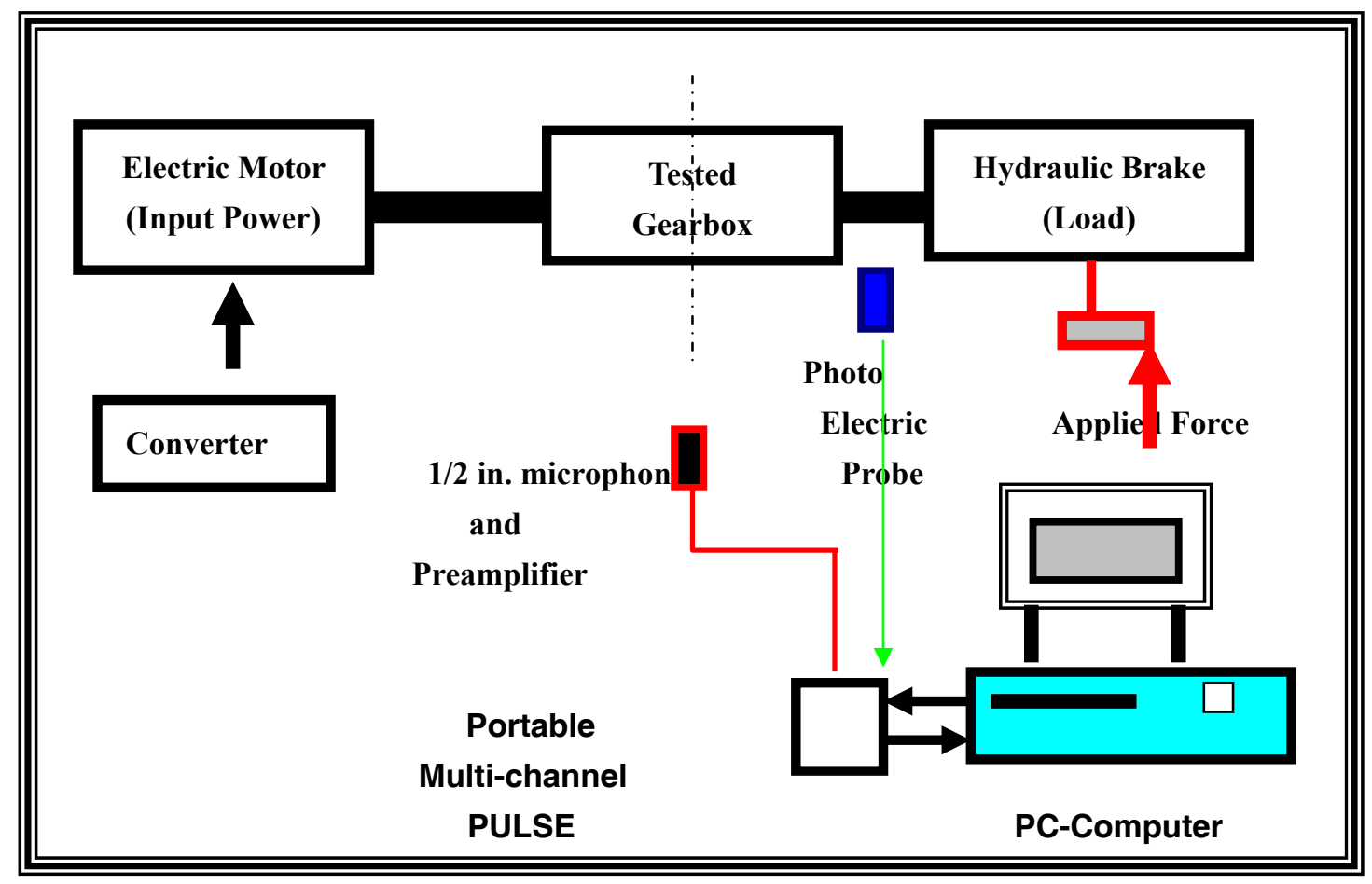

Figure 4. Experimental setup

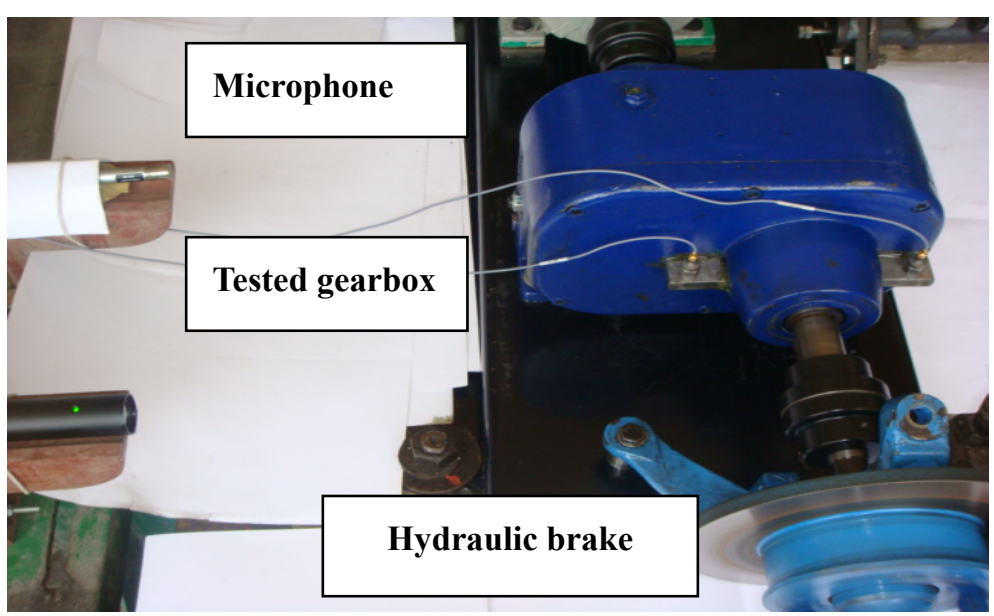

Figure 5. Sound pressure level (SPL) measurement 

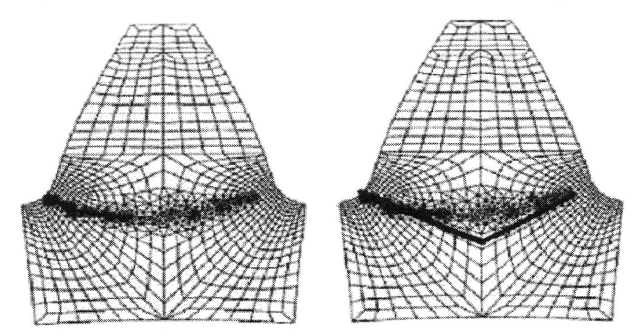

Figure 6. Assumed crack propagation path

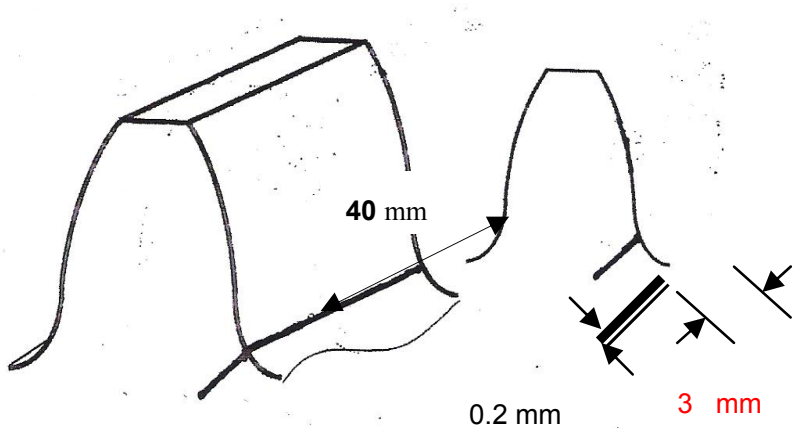

Figure 8. Sketch of the crack dimensions

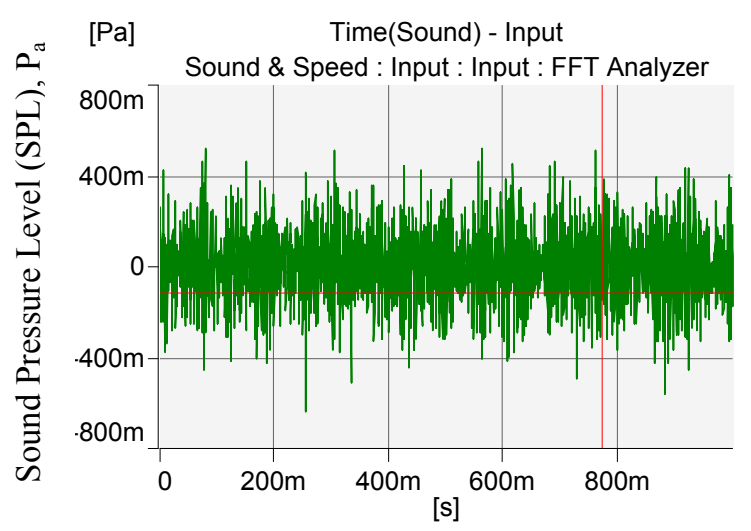

Figure 10. Measured SPL crack depth $3.00 \mathrm{~mm}$, testing time $0.0 \mathrm{~h}$, speed $400 \mathrm{rpm}$, load $15 \mathrm{Nm}$

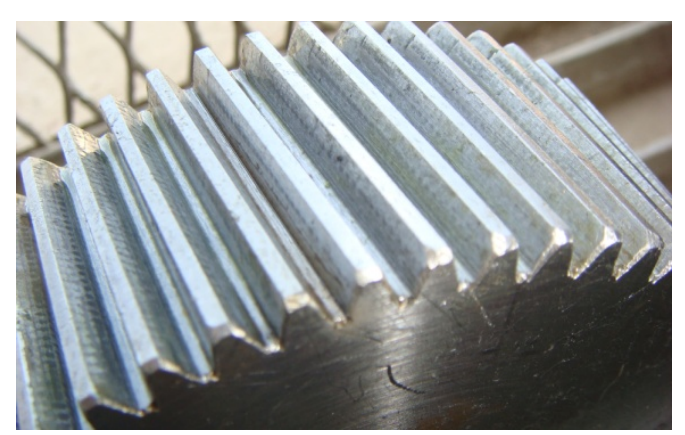

Figure 7. Gear in health condition

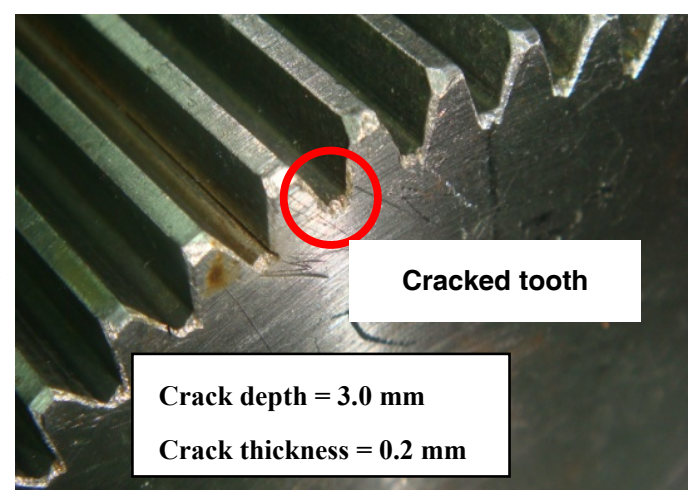

Figure 9. Gear defected (cracked

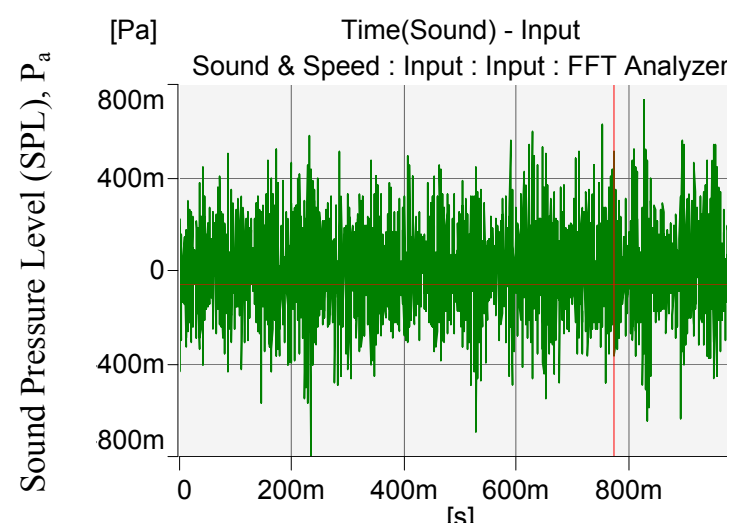

Figure 11. Measured SPL crack depth $3.00 \mathrm{~mm}$, testing time $6.0 \mathrm{~h}$, speed $400 \mathrm{rpm}$, load $15 \mathrm{Nm}$ 


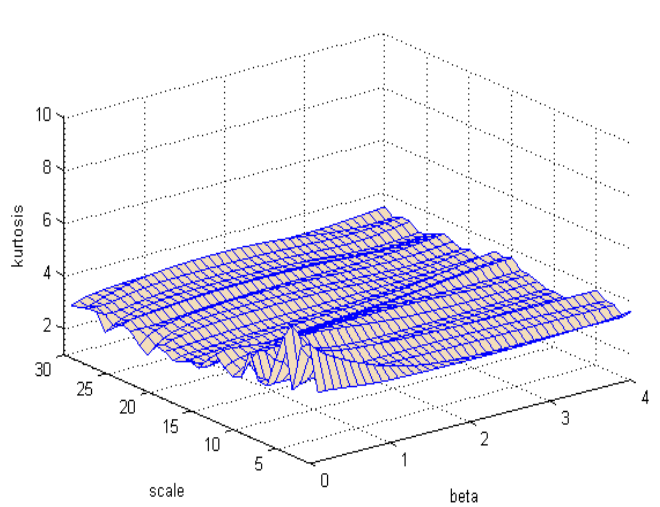

Figure 12. The kurtosis distribution for different values of Scale (a) and values of beta ( $\beta$ ), $400 \mathrm{rpm}$, Load $15 \mathrm{Nm}$, Crack depth $3 \mathrm{~mm}$
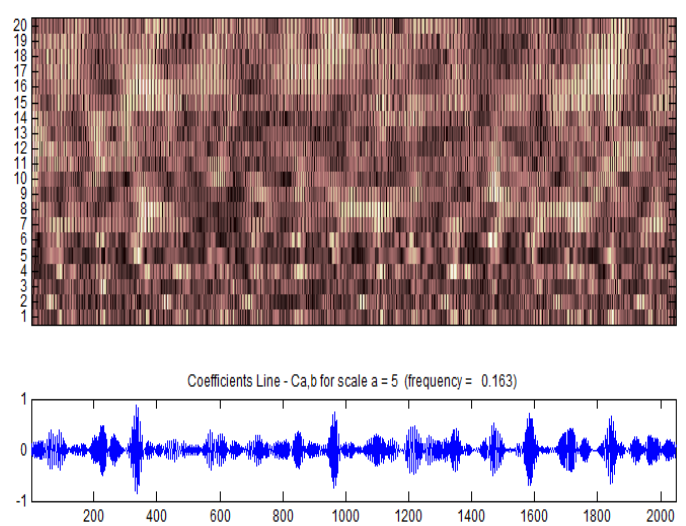

$$
\text { Beta }=0.2, \text { Scale }=5
$$

Testing time $6.0 \mathrm{~h}, 400 \mathrm{rpm}$, load $15 \mathrm{Nm}$, crack depth $3 \mathrm{~mm}$ Figure 14. Wavelet map of SPL Morlet wavelet signals for faulty (cracked) gear

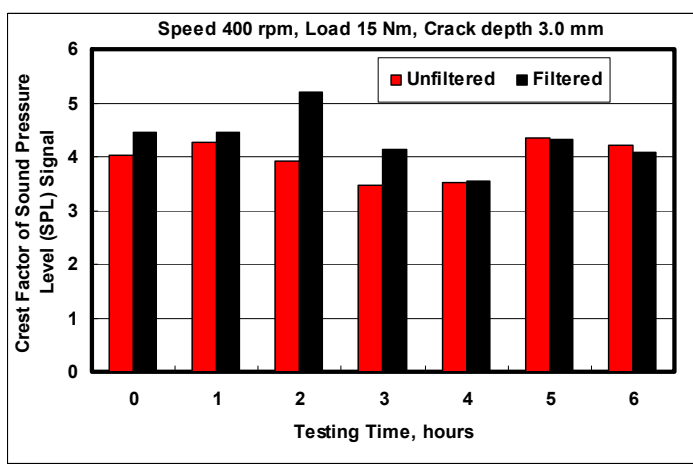

Figure 16. Crest factor for sound pressure level (SPL) - Faulty (cracked) gear

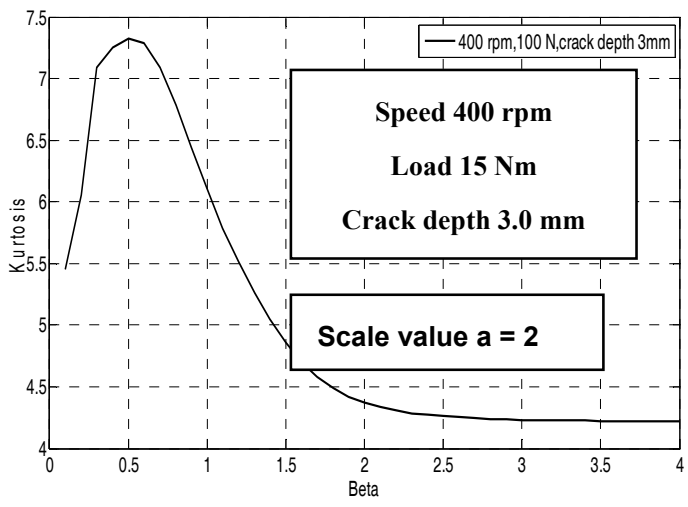

Figure 13. Kurtosis as function of beta $(\beta)$

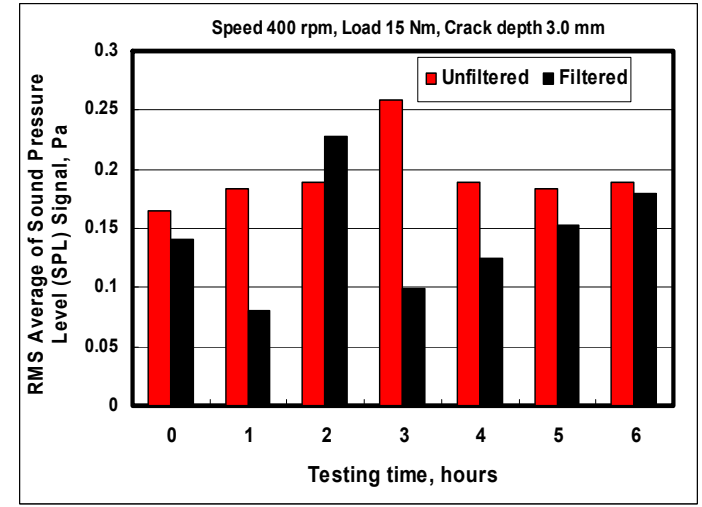

Figure 15. RMS Sound pressure level (SPL) for faulty (cracked) gear

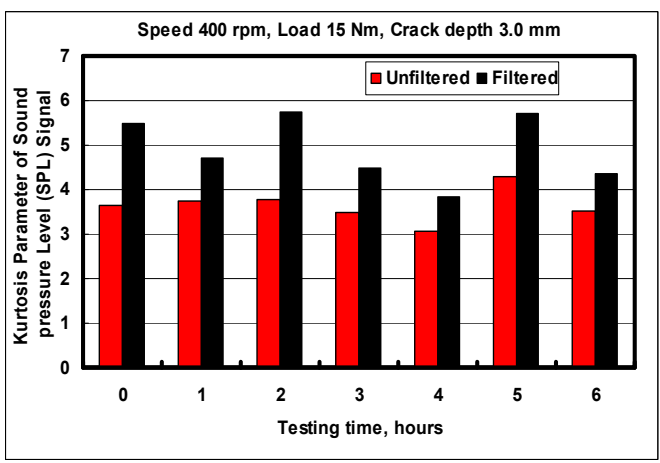

Figure 17. Kurtosis parameter for sound pressure level (SPL) - faulty (cracked) gear 


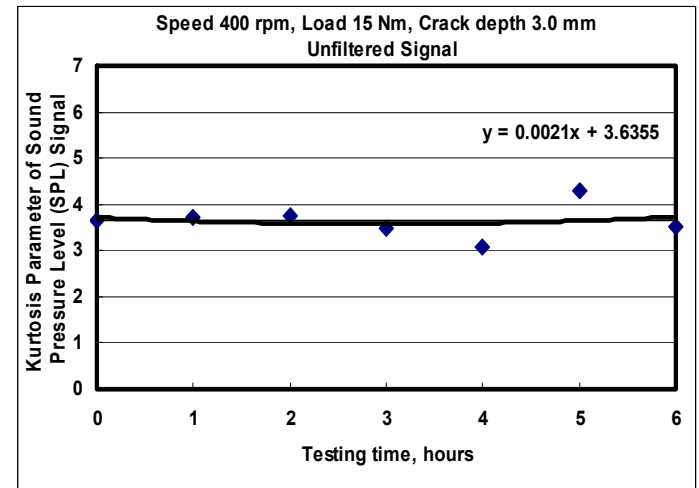

Figure 18. Kurtosis parameter for sound pressure Level (SPL) - faulty (cracked) gear

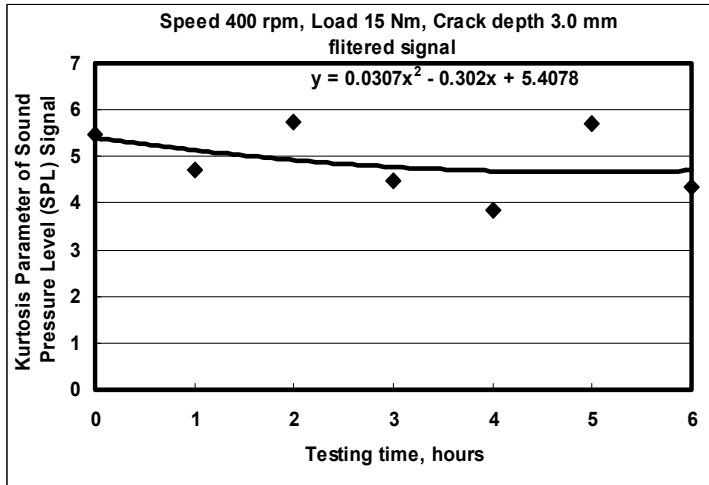

Figure 19. Kurtosis parameter for sound pressure Level (SPL) - faulty (cracked) gear

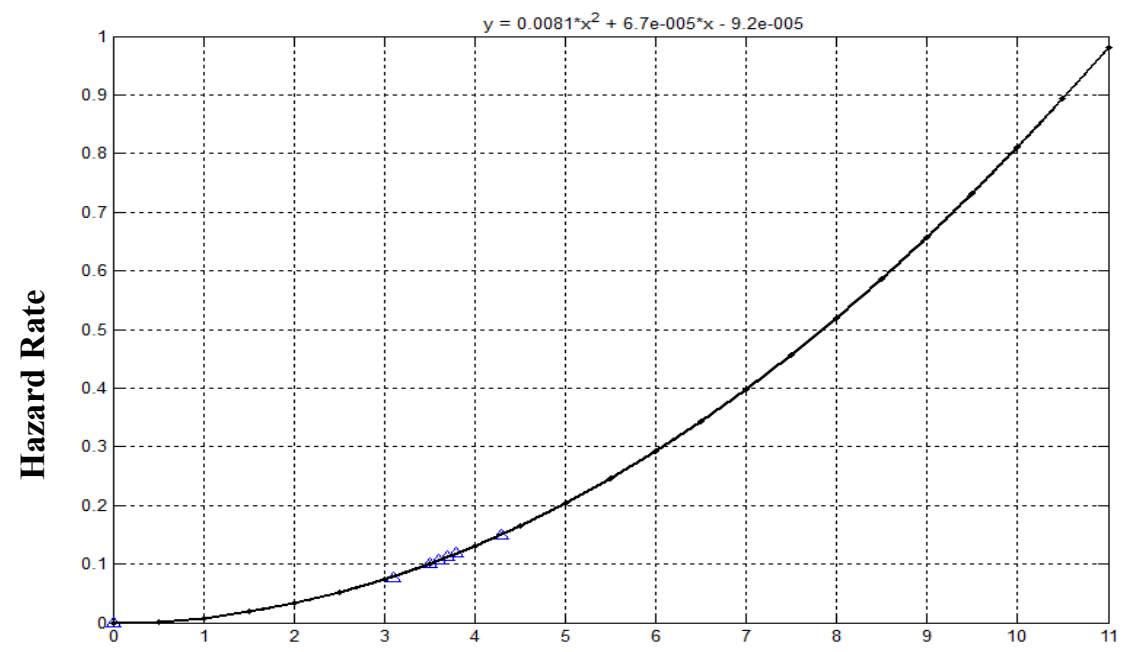

Figure 20. Relationship between hazard rate and Kurtosis in unfiltered form (actual data)

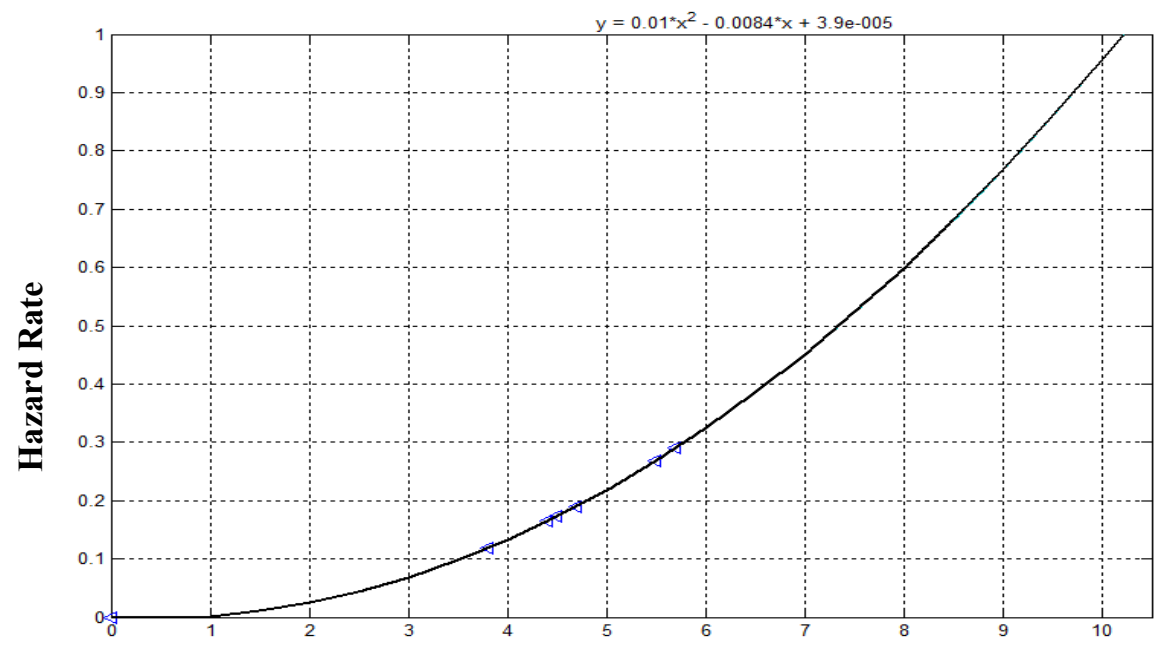

Figure 21. Relationship between hazard rate and Kurtosis in filtered form (actual data) 


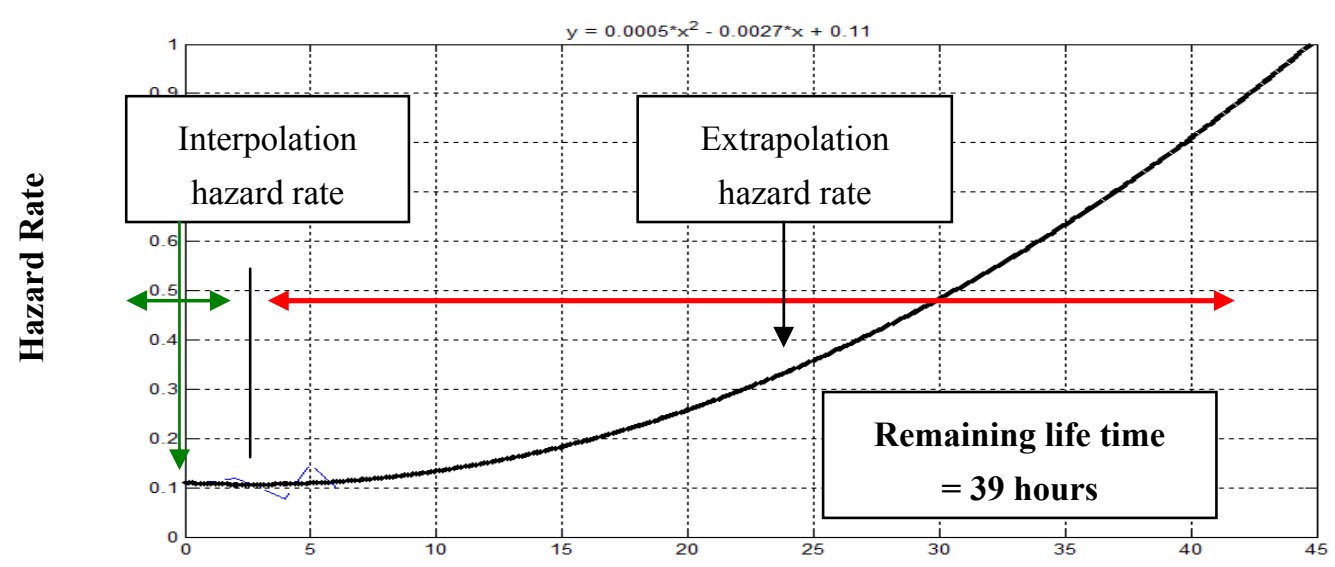

Time, hours

Figure 22. Remaining life time for unfiltered signal

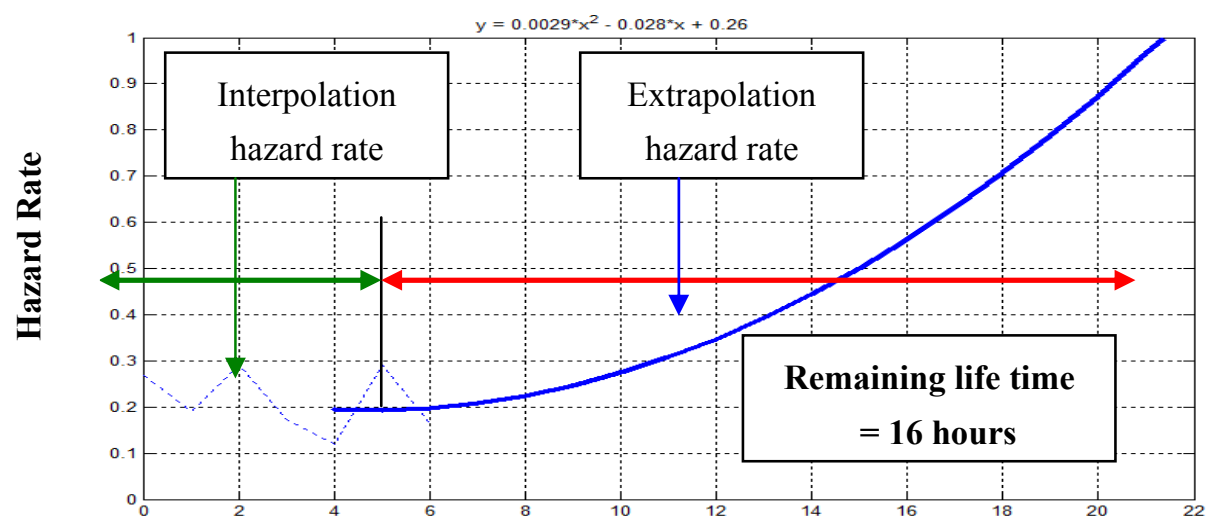

Time, hours

Figure 23. Remaining life time for filtered signal 